\title{
CBiOS Science Sessions Proceedings -2012 -
}
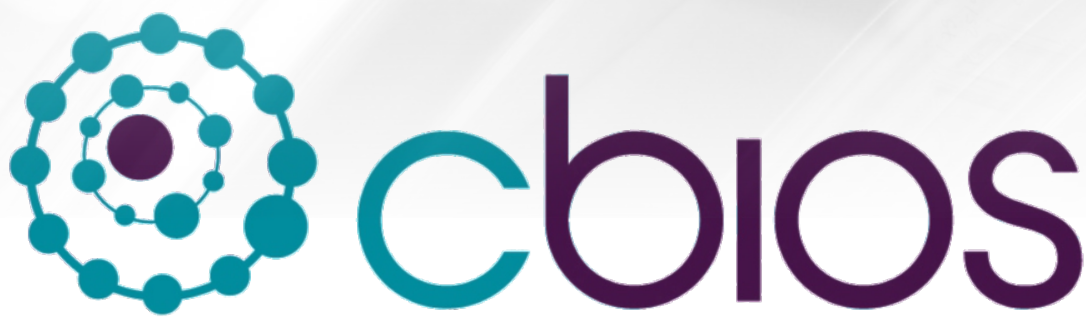

UNIVERSIDADE LUSÓFONA RESEARCH CENTER FOR HEALTH SCIENCES \& TECHNOLOGIES

Study of the potential applicability of the by-products of the Prunus cerasus in promoting health and skin care Elisabete Maurício, Catarina Rosado, A. Diaz

\section{9}

Contribution to a biologic and legal framework of the M. sylvestris in skin repair

Graziela Sponchiado, Cristina Mello-Sampayo, Maria M. Pereira ${ }^{4}$, Henrique Silva, Michel Otuki , Beatriz Silva-Lima, and Luís M. Rodrigues

240

Nanotechnology and drug delivery systems

\section{Catarina Pinto Reis}

241

Bacterial cellulose membranes applied in topical and transdermal delivery

Eliane Trovatti, Nuno Silva, Carmen Freire, Armando Silvestre, Catarina Rosado

New treatment strategies with plant extract teas for Alzheimer's disease 


\title{
Study of the potential applicability of the by-products of the Prunus cerasus in promoting health and skin care
}

\author{
Elisabete Maurício ${ }^{1,2}$, Catarina Rosado $^{1}$, A. Diaz $^{2}$ \\ ${ }^{1}$ CBIOS - Research center for Health Sciences \& Technologies, U. Lusófona, Campo Grande 376, \\ 1749-024 Lisboa, Portugal \\ ${ }^{2}$ Pharmacology Unit, Alcalá de Henares University, Alcalá de Henares, Spain
}

\begin{abstract}
In recent years many studies on cherries have revealed that they are rich sources of bioactive compounds, mainly due to its polyphenolic phytochemicals. In this work, by-products of the sour cherry (Prunus cerasus L.) used in the Obidos liquor from Portugal, were evaluated by determination of their phenolic content and antioxidant activity. The samples (stems, leaves and pomace) were extracted by maceration with different solvents (acetone, ethanol, methanol and water). The optimal extraction time was determinated during 30 hours. All the extracts with solvents were evapored or liofilized and suspended in glycol or water. The Total Phenolic Content was determined with the Folin-Ciocolteau method and the antioxidant activity was confirmed by determination of their free radical scavenging activity through DPPH and FRAP assay.The best extraction time observed for the pomace extracts was 12 hours and for the leaves and stems 24 hours. The best solvent was methanol for all the samples .Stems extracts showed a higher concentration in polyphenols and antioxidant activity than those from leaves and pomace extracts. Further studies will be conducted to address their potential use as dermocosmetic ingredients.
\end{abstract}

\section{Lecturer's resumé}

Elisabete Mauricio is currenthy Technical Director at Elisa Câmara, Lda, a dermocosmetic Company where she is responsible for the Development and Research of Natural Cosmetic formulations, since 1995. She is assistant professor in the Faculty of Engineering at Universidade Lusófona, lecturing Microbiology and Cellular Biology, since 1996. She is also Consultant for Microbiological and Toxicology Safety Assessment for Cosmetics and Body Care products recognized by Infarmed since 2001. Elisabete is a PhD student at University of Alcalá, Spain in association with CBIOS and UDE at Universidade Lusófona. She attended New University of Lisbon, Faculty of Sciences and Technology for her graduation (1993) in Engineering, as well as in her and Master degree (2003) in Food Technology/Chemistry. In the same faculty she attended the Post-Graduation program in Management of Bio-Chemical Analysis Laboratories (1995). In 2006/2007 Elisabete completed the Post-Graduate program in Dermocosmetic Sciences at Universidade Lusófona. 
)

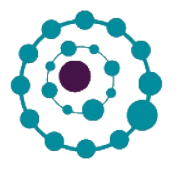

\title{
Contribution to a biologic and legal framework of the M. sylvestris in skin repair
}

\author{
Graziela Sponchiado ${ }^{1,2,4}$, Cristina Mello-Sampayo ${ }^{1}$, Maria M. Pereira ${ }^{4}$, Henrique Silva ${ }^{3,4}$, Michel Otuki ${ }^{2,5}$, \\ Beatriz Silva-Lima ${ }^{1,3}$, and Luís M. Rodrigues ${ }^{3,4}$ \\ ${ }^{1}$ iMed Research Institute for Medicines and Pharmaceutical Sciences, Universidade de Lisboa - Fac Pharmacy, \\ Lisbon, Portugal \\ ${ }^{2}$ Universidade Federal de Paraná (UFPR), Curitiba, PR, Brazil \\ ${ }^{3}$ Pharm. Sciences Department - Universidade de Lisboa - Faculty of Pharmacy, Lisboa, Portugal \\ ${ }^{4}$ CBIOS Universidade Lusófona, Lisboa, Portugal; ${ }^{5}$ Department of Pharmaceutical Sciences, State University of \\ Ponta Grossa (UEPG), Ponta Grossa, PR, Brazil.
}

\begin{abstract}
Treatment with medicinal plants were for centuries your sole remedy available, and today still generate a great interest of the pharmaceutical industry as a source of new medicines, especially by the need for innovation in the treatment of some diseases, but there are no studies that prove the biological activity of these natural substances. The Malva sylvestris consumption has reported since $3000 \mathrm{BC}$ but without much proof of efficacy and safety, thus showing the need to raise the level of scientific evidence about its use, such as in skin inflammatory processes. The study aims to develop a minimal animal (Wistar rat) model of skin recovery, less aggressive and that can also be used in the translation to the human model. The although preliminary results obtained by the biometric variables non invasives measuring involving transepidermal water loss (TEWL, Tewameter TM300), erythema (Chromameter $\left.{ }^{\circledR}\right)$ and local microcirculation (LDF-Periflux ${ }^{\circledR}$ ), are being positive when used as a chemical challenge SLS (sodium lauryl sulphate) in low concentrations ( $2 \%$ e $3.5 \%)$. Even though the animal model developed can be used for various applications in skin physiology is still needed to optimize some details on the methodology before start to the next stage, tests with the extract of M. sylvestris.
\end{abstract}

\section{Lecturer's resumé}

Graziela Sponchiado is currently a of Pharmaceutical Sciences Postgraduate Program $\mathrm{PhD}$ grantee, at the Federal University of Paraná (UFPR), Curitiba, Brazil, by the Program Doctoral Sandwich Abroad (PDSE) CAPES (Coordination for the improvement of Higher Education Personnel) - Brazil, in iMed Research Institute for Medicines and Pharmaceutical Sciences, University de Lisboa - Fac Pharmacy and in CBIOS - Research Center for Health Sciences \& Technologies, University Lusófona, Lisboa, Portugal.

She graduated in Biological Sciences (2002) at the University Positivo (Curitiba, PR - Brazil) where she concluded her master's degree studies in Environmental Management (2008). 


\title{
CBiOS Science Sessions - 2012
}

\author{
Nanotechnology and drug delivery systems
}

\author{
Catarina Pinto Reis \\ CBIOS - Universidade Lusófona's Research Center for Health Science and Technologies, \\ Campo Grande, 376, 1749-024, Lisboa, Portugal
}

\begin{abstract}
Nanotechnology is unique in that it represents not just one specific area but a vast variety of areas ranging from basic material science to medical fields.

The concept of nanoparticles for the design of novel drug delivery systems emerged a few years ago and recent rapid advances in nanotechnology have offered a wealth of new opportunities for diagnosis and therapy of various diseases. However, it is a fair and important question to ask when nanotechnology will realistically bring clinically useful drug delivery systems? Hopefully soon but theorically it should have happened already since several nanoproducts are on the market and a lot more is coming for development pipeline. Unfortunately, there are still some issues to overcome. Firstly, the pharmaceutical industry has been slow to use this novel drug delivery sustems if they include componentes that are not classified as GRAS by FDA. Secondly, there is some resistance in terms of adding any untested materials that may require seeking approval of the nanoproduct. Finally, in general, the new entity is a long and costly process. To overcome those problems, several research groups (as our) are developing new drug delivery systems that are substantially better that existing delivery systems and unlike many strategies, nanotechnology has a potential just do to that: increases in efficacy by orders of magnitude. The future of nanotechnology-based drug delivery systems only depends on the ability to scale-up production by nano manufacturing.
\end{abstract}

\section{Lecturer's resumé}

Catarina Pinto Reis is currently Professor of Pharmaceutical Technology in the Faculty of Sciences and Health Technologies at the Universidade Lusófona de Humanidades e Tecnologias (ULHT). She is the Director for Innovation of CBIOS and coordinator of Laboratory of Nanoscience and Biomedical Nanotechnology at ULHT. She is also a consultant for the National Authority of Medicines and Health Products (INFARMED).

She attended the University of Coimbra (Coimbra, Portugal) and graduated with First Class Honours in Pharmaceutical Sciences in 2003. Her doctoral studies were also undertaken at the University of Coimbra with several important international collaborations where she developed an interest in advanced drug delivery systems. Those studies have been recognized through a number of national prizes. She finished her $\mathrm{PhD}$ in Pharmacy as Distinguished Honor Graduate. Since then, she has continued to work in the design and evaluation of nanoparticles and microparticles for peptide drug and gene delivery. She is author or co-author of several articles, book chapters and inventor of patents. 


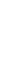

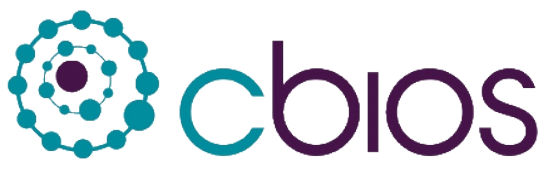

\section{CBiOS Science Sessions - 2012}

\section{Bacterial cellulose membranes applied in topical and transdermal delivery}

\author{
Eliane Trovatti ${ }^{1}$, Nuno Silva ${ }^{1}$, Carmen Freire ${ }^{1}$, Armando Silvestre ${ }^{1}$, Catarina Rosado ${ }^{2}$ \\ ${ }^{1}$ CICECO and Department of Chemistry, University of Aveiro, Aveiro, Portugal \\ ${ }^{2}$ CBIOS/ Experimental Dermatology Unit, Lusófona University, Lisbon, Portugal
}

\begin{abstract}
Bacterial cellulose $(\mathrm{BC})$ is a biomaterial with unique physical and mechanical properties that triggered considerable interest, but there are few studies addressing the use of such membranes for drug loading and controlled release. This study aimed to investigate the applicability of BC membranes in topical or transdermal drug delivery systems. To assess its therapeutic feasibility, the permeation through human epidermis of model drugs (lidocaine hydrochloride, ibuprofen, caffeine and diclofenac) in $\mathrm{BC}$ and other formulation systems was compared in vitro.

Diffusion studies with Franz cells showed that the incorporation the drugs in BC membranes provided similar steady-state fluxes to those obtained with the conventional formulations.

These results indicate that this technology can be successfully applied to modulate the bioavailability of drugs for percutaneous administration, which could be particularly advantageous in the design of delivery systems that have, simultaneously, the ability to absorb exudates and to adhere to irregular skin surfaces.
\end{abstract}

\section{Lecturer's resumé}

Catarina Rosado was educated in the Faculty of Pharmacy of the University of Coimbra, where she obtained her degree in Pharmaceutical Sciences in 1997. In 2000 she completed her PhD at Cardiff University.

She returned to Portugal in 2001 and initiated a Post-Doc. In 2004, she joined the faculty of the Universidade Lusófona as a lecturer of the Faculty of Health Sciences. She has established her research in the Experimental Dermatology Unit, but has established collaborations with other Portuguese universities. Her research interests focus mainly on the study of the impact of formulation on transdermal penetration and also in the development of non-invasive in vivo strategies to assess efficacy and safety of topical drugs and cosmetics.

Catarina Rosado is the director of the MSc program in Dermato-Cosmetic Sciences of the Universidade Lusófona. She has regularly been invited to lecture in Masters and Postgraduate programs in other Portuguese universities. 


\begin{abstract}
Acetylcholinesterase (AChE) is the enzyme that catalyzes the hydrolysis of the neurotransmitter acetylcholine. Currently, the most successful therapy for Alzheimer's disease (AD) consists of increasing the levels of acetylcholine through the inhibition of AChE activity. In this study, the in vitro AChE inhibition, antioxidant activity and bioactive components of Plectranthus grandidentatus, P. porcatus, P. ornatus, P. ecklonii and P. saccatus aqueous extracts were investigated and compared by four extraction methods (infusion, decoction, microwave and ultrasonic extractions). The amounts of dry plant extracts were obtained and P. ecklonii gave the highest yield. The main components rosmarinic, chlorogenic and caffeic acids were quantified by HPLC-DAD for each extraction method. The decoctions showed high AChE inhibition and antioxidant activities (P. ecklonii and P. saccatus) and microwave extractions showed higher inhibition activity (P. grandidentatus and P. ornatus). To increase stability and control extract release, microwave P. ecklonii extract was encapsulated into alginate beads (encapsulation efficiency range $=98.64-100.0 \%$ ). The evaluation of bioactivity IC50 values, microspheres and nanoparticles production are under study.
\end{abstract}

\title{
Lecturer's resumé
}

Patrícia Rijo is currently an Assistant Professor at the Faculty Sciences and Technologies of Health at the Universidade Lusófona de Humanidades e Tecnologias; Escola Superior de Saúde Ribeiro Sanches (Lisbon, Portugal) since 2001 and member of Phytochemical Society of Europe and Sociedade Portuguesa de Fitoquímica e Fitoterapia. She attended the University of Lisbon at the Faculdade de Ciências for her graduation and Master degree and PhD at Faculdade de Farmácia. She has several papers and communications in international peer-reviewed journals and meetings and works in Natural Products Chemistry since 1998. Considering her research interest in Natural Products she currently collaborates with Research Institute for Medicines and Pharmaceuticals Sciences - iMed.UL, Centro de Química e Bioquímica FCUL Free Radicals, Health and Nutrition group Centro de Biotecnologia Vegetal (IBB)-FCUL and Departamento de Química Bio-Orgánica Instituto de Química Orgánica General, CSIC, Madrid. 